Research Article

\title{
Traceability Management Strategy of the EV Power Battery Based on the Blockchain
}

\author{
Yanjin Cheng $\left(\mathbb{D}\right.$, Hao Hao ${ }^{D}$, Shipeng Tao $\mathbb{D}$, and Yanjun Zhou \\ School of Economics and Management, Shanghai Polytechnic University, Shanghai 201209, China \\ Correspondence should be addressed to Hao Hao; haohao@sspu.edu.cn
}

Received 20 April 2021; Revised 8 July 2021; Accepted 11 August 2021; Published 24 August 2021

Academic Editor: Tingsong Wang

Copyright ( 2021 Yanjin Cheng et al. This is an open access article distributed under the Creative Commons Attribution License, which permits unrestricted use, distribution, and reproduction in any medium, provided the original work is properly cited.

\begin{abstract}
Regulating and supervising the energy vehicle (EV) power battery recycling market, improving the utilization rate of EV power battery recycling, and guaranteeing the safety and control of all aspects of recycling treatment require the establishment of an effective traceability system. The decentralization and tamper-proof characteristics of the blockchain can ensure the safety and reliability of relevant data while realizing traceability management. This study establishes the Stackelberg game model to compare and analyze the effects of different government mechanisms on the profits of each subject before and after participating in power battery traceability management. The study further uses the model to explore strategies to improve the enthusiasm of EV power battery recycling subjects to participate in traceability management. The results show that (1) the participation of each recycling subject in EV power battery blockchain traceability can help move more spent power batteries into formal recycling channels; (2) the government should adopt appropriate mechanisms to promote its participation in EV power battery blockchain traceability, the best result being when the government adopts a subsidy mechanism for consumers; and (3) the profit of the EV power battery manufacturer is inversely proportional to the target recycling rate set by the government. Furthermore, the pursuit of a very high target recycling rate is not conducive to the normal implementation of initial EV power battery blockchain traceability management. Therefore, it is crucial for the government to set a reasonable target recycling rate.
\end{abstract}

\section{Introduction}

In recent years, China's environmental pollution problem has become increasingly serious, with frequent transportation activities emitting large amounts of pollutants and becoming a major source of environmental pollution and energy consumption [1]. In 2010, the Chinese government listed the "new energy" and "new energy vehicle (NEV)" industries as national strategic emerging industry sectors to accelerate the development of the renewable energy industry and solve the environmental pollution and energy crises [2]. According to the data released by the China Association of Automobile Manufacturers (CAAM), the production and sale of the NEV in China reached 1.366 and 1.367 million units, respectively, in 2020. Of these, 1.105 million and 1.115 million units of energy vehicles (EVs) were produced and sold, with a yearly growth of $5.4 \%$ and $11.6 \%$, respectively [3]. The use of power batteries has increased considerably following the continuous development of the EV industry. The 5 to 8 years of use of EV power batteries show that China entered the peak of power battery end of life around 2020 [4]. The improper disposal of spent EV power batteries not only increases the risk of environmental pollution but also leads to the wastage of precious metal resources [5]. Today's deteriorating ecological environment and depleted natural resources and EV power battery recycling-related enterprises and organizations proactively carry out the entire life cycle supply chain management of power batteries to realize the recycling and resource utilization of $\mathrm{EV}$ power batteries. These can yield huge economic and ecological benefits $[6,7]$. Companies obtain more value from the reverse logistics and remanufacturing process of spent EV power batteries while also protecting the ecological environment [8].

China began recycling spent EV power batteries later than other developed countries; however, the development status is not optimistic. Moreover, the recycling and 
government regulatory systems need to be improved [9]. First, informal recycling channels coexist with formal recycling channels. The recycling methods of informal recycling channels are more convenient. Therefore, a large number of spent EV power batteries flow into informal recycling channels. Furthermore, it is difficult for formal recyclers to form large-scale benefits. Second, the synergy of the subjects of production-recycling-echelon use is weak. Moreover, there are data and technical barriers between government departments and other enterprises. These make it more difficult to recycle and process spent EV power batteries as well as supervise each recycling subject.

To regulate the recycling market order of spent EV power batteries, the Ministry of Industry and Information Technology (MIIT) issued the "Interim Regulations on the Traceability Management of the Recycling and Utilization of New Energy Vehicle Power Batteries." This proposed the requirements for the establishment of "the Integrated Management Platform for the National Monitoring of New Energy Vehicles and Traceability of Power Battery Recycling," hoping to realize the information collection and supervision of the entire process of power battery production, sales, use, end of life, recycling, and utilization [10]. Furthermore, the traceability management of EV power batteries in China entered the primary stage. However, the existing EV power battery traceability system cannot guarantee a high level of system reliability, data accuracy, and information transparency. Moreover, some EV power battery manufacturers and formal recyclers are not very active in EV power battery traceability management considering issues such as data privacy and security. Additionally, China's policies and regulations in this field still need to be improved. These result in the slow promotion of China's EV power battery traceability management.

Blockchain technology is characterized by immutability and distributed storage among other characteristics. The data security problem of the power battery traceability system can be solved using computer technologies such as point-to-point transmission, consensus mechanism, and encryption algorithm [11]. After the data are deposited into the blockchain to obtain a consensus of the network nodes, it is difficult to be tampered with, which ensures the authenticity and security of the information related to the power battery [12]. Blockchain can realize the transparency of the data of the entire process of the EV power battery supply chain by using a consensus mechanism, improving the knowledge of government departments and related enterprises on the entire process of power battery recycling and breaking the data barriers, so as to further realize source traceability, process supervision, and risk early warning. The government should simultaneously adopt certain mechanisms to encourage EV power battery recycling and treatment-related enterprises to join this traceability management platform. Therefore, this study aims to explore strategies to improve the enthusiasm of EV power battery recycling subjects to further participate in traceability management by establishing the Stackelberg game model. The game model is of great significance in promoting the highquality development of the power battery recycling industry.
The potential contributions of the study include the following:

(1) Based on summarizing the research of domestic and foreign scholars in the field of EV power battery recycling, the study innovatively proposed the application of blockchain traceability management to EV power battery recycling, hence filling the research gaps in this research field.

(2) Considering different government guidance mechanisms, a comparative analysis of the influence of government rewards, punishments, and subsidies on the enthusiasm of power battery recycling subjects to participate in traceability management is analyzed; government departments and power battery recycling and processing companies provide decisionmaking references.

(3) The Stackelberg game model was established considering factors such as consumer willingness and blockchain cost. An empirical analysis verified the effectiveness of the model.

The rest of this paper proceeds as follows. Section 2 introduces the current research status. Section 3 describes the model. Section 4 compares and analyzes the optimal situation under different strategies. Section 5 uses relevant data for empirical research. In conclusion, Section 6 summarizes and discusses the limitations of this research.

\section{Literature Review}

Currently, research on recycling channels and EV power battery recycling in the domestic and foreign literature mainly involves the selection of recycling channels, pricing decisions under multichannel competition, and the influence of government coordination mechanisms. Research on blockchain traceability management mainly focuses on food and medical care. Furthermore, research in the field of EV power battery recycling is scarce.

Regarding research on recycling channels, Savaskan et al. [13] compared and analyzed three recycling models with manufacturers, distributors, and third-party recyclers as recycling takers. They found that the recycling model with distributors as recycling takers was optimal, all else being equal. Liu et al. [14] analyzed the impact of government subsidies on formal and informal recycling channels while considering the quality of the recycled products. They found that the marginal effect of government subsidies was insignificant when the quality of the recycled products was high. Li et al. [15] developed a Stackelberg game model with dual recycling channels while considering consumer preferences. They concluded that the improvement of environmental benefits under government governance mechanisms depends on subsidies and consumer channel preferences. Tang et al. [16] used social welfare as an indicator for selecting recycling models and built Stackelberg game models under three single-recycling channel models and three competitive dual-recycling channel models based on the government's reward and penalty mechanism. They 
found that setting a reasonable minimum power battery recycling rate as a benchmark for the reward and penalty mechanism is crucial. Xia et al. [9] studied the impact of competition between mixed WEEE sales channels and recycling channels on CLSC decisions under the government mechanism. They found the optimal strategy of each company and government based on the Stackelberg game model.

Regarding research on power battery recycling, owing to the increasing depletion of natural resources, the recycling of products and materials is a growing concern [17]. Research in this field can be categorized into two types: qualitative and quantitative. Regarding the qualitative aspects of power battery research, Wang and $\mathrm{Wu}[18]$ analyzed the challenges in the recycling process of $\mathrm{LiFePO}_{4}$ batteries in China and proposed improvement strategies to make the recycling chain more environmentally friendly. Zeng et al. [5] analyzed the potential environmental and safety risks in the recycling and disposal processes of used lithium batteries and proposed a comprehensive management scheme regarding policies and regulations, recycling systems, and recycling technology. Beaudet et al. [19] analyzed the main challenges and opportunities for recycling new energy vehicle batteries and made relevant recommendations to address these challenges. Hao et al. [20] combined the current status of reverse logistics of power battery recycling and proposed effective countermeasures to contribute to the development of reverse logistics of power battery recycling. Regarding the quantitative aspects of power batteries, $\mathrm{Li}$ et al. [21] studied the coordination strategies of each supply chain subject in a three-tier reverse supply chain consisting of recyclers, remanufacturers, and retailers and conducted a detailed comparative analysis of the optimal decisions under different strategies. Gu et al. [22] studied the optimal pricing strategy for the closed-loop supply chain of power batteries and found that recycling spent power batteries does not benefit the profits of power battery manufacturers. They further suggested some government incentives to improve the economic benefits of recycling. Tang et al. [23] explored the social, economic, and environmental impacts of recycling end-of-life electric vehicle batteries under government subsidies and reward and punishment mechanisms, respectively. Hao et al. [17] established an improved fuzzy neural network evaluation structure model based on the combination of the fuzzy comprehensive evaluation method and particle swarm algorithm- (PSO-) optimized error backpropagation (BP) neural network with the feasibility of reverse logistics of end-of-life automotive power batteries as the target layer. Wang et al. [24] considered the recycling and remanufacturing costs of waste batteries and carbon tax to establish the lowest total cost mixed-integer programming problem model for the recycling network of NEVs. Wang et al. [25] analyzed the impact of multiattribute decisionmaking (MADM) on the efficiency of the end-of-life vehicle (ELV) reverse logistics industry in the context of a circular economy, hence indirectly providing a basis for management and investment decisions in the new energy vehicle power battery recycling industry. It is beneficial to explore the recycling of waste to promote the construction of ecological civilization against the background of a comprehensive implementation of the "Healthy China" strategy [26].

Research in blockchain traceability management focuses on food and medical care. Dandage et al. [27] analyzed the necessity of traceability management in the food industry and introduced the relevant technologies used with the help of traceability management. Tsang et al. [28] and Lin et al. [29] addressed the problems of opaque information, easy tampering, centralization, and serious information silos in traditional food traceability systems. Caro et al. [30] and Feng et al. [31] proposed a food safety traceability system based on the blockchain and Internet of Things (IoT) based on the analysis of the problems of traditional agricultural product supply chain traceability systems. Gong et al. [32] built a blockchain-based power battery data sharing system for real-time data monitoring and the sharing of the power battery life cycle. Gopalakrishnan et al. [33] proposed a blockchain-based solid waste management (SWM) model to solve the problem of waste traceability. Furthermore, the SWM system with the cost element of the blockchain was optimized.

Through the aforementioned combination of the domestic and foreign research literature, it is evident that various research scholars have studied the selection of recycling channels and EV power battery recycling from different perspectives; however, research on EV power battery traceability management in the context of blockchain is relatively rare. Based on the lack of research in this area, this study examines the blockchain-based EV power battery traceability management strategy and analyzes the influence of different government mechanisms on the unit recycling price, quantity, and profit of each subject. We hope to fill the research gap in this field and provide a relevant basis for the formulation of relevant policies and regulations as well as a reference for the decision-making of EV power battery recycling-related enterprises.

\section{Model Description and Construction}

3.1. Problem Description. Data barriers and technical barriers exist between EV power battery recycling subjects and government enterprises, which make it more difficult for enterprises to recycle spent $\mathrm{EV}$ power batteries and for the government to supervise each recycling subject. The Ministry of Industry and Information Technology requires the establishment of a power battery traceability platform, so as to realize the information collection of the whole process of power battery production, use, end of life, recycling, and utilization. It can also realize the implementation of the monitoring of the responsibility of each link subject to fulfill the recycling. However, the existing EV power battery traceability system for energy vehicles cannot guarantee a high level of system reliability and data security, enterprises are not highly motivated to participate in power battery traceability management, and China's policies and regulations in this field still need to be improved, resulting in the slow promotion of EV power battery traceability management in China. Blockchain technology is characterized by 


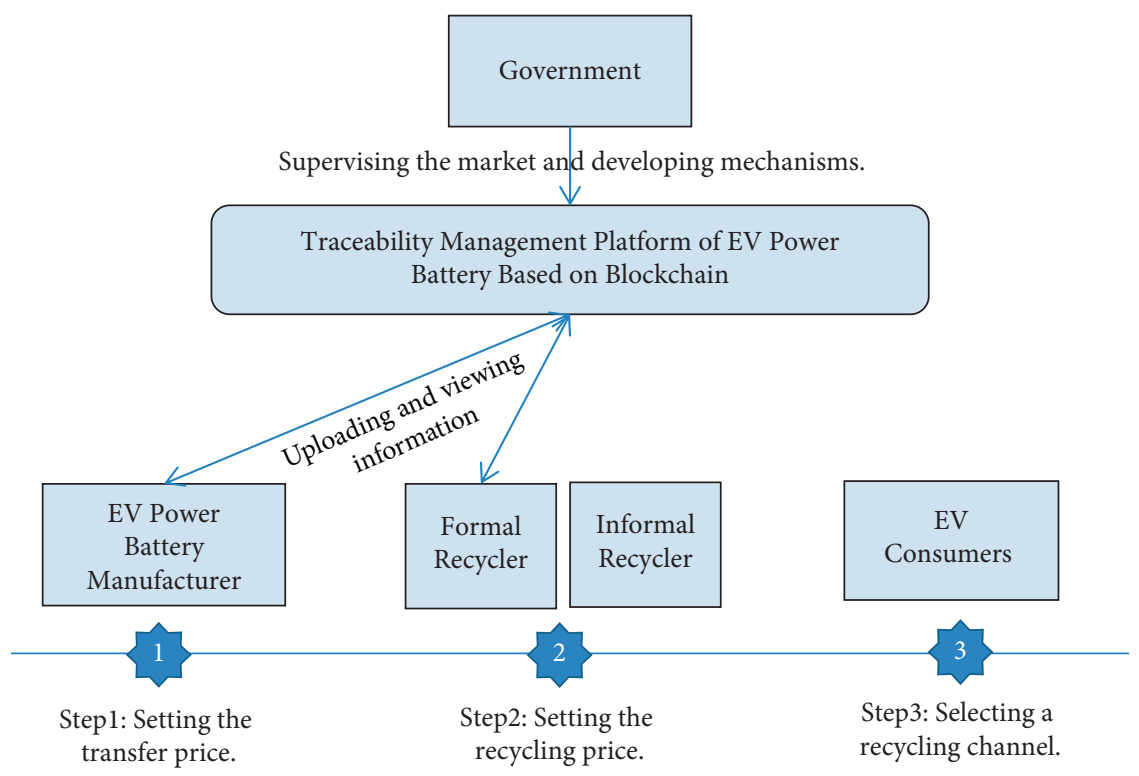

Figure 1: Topological structure diagram of the model.

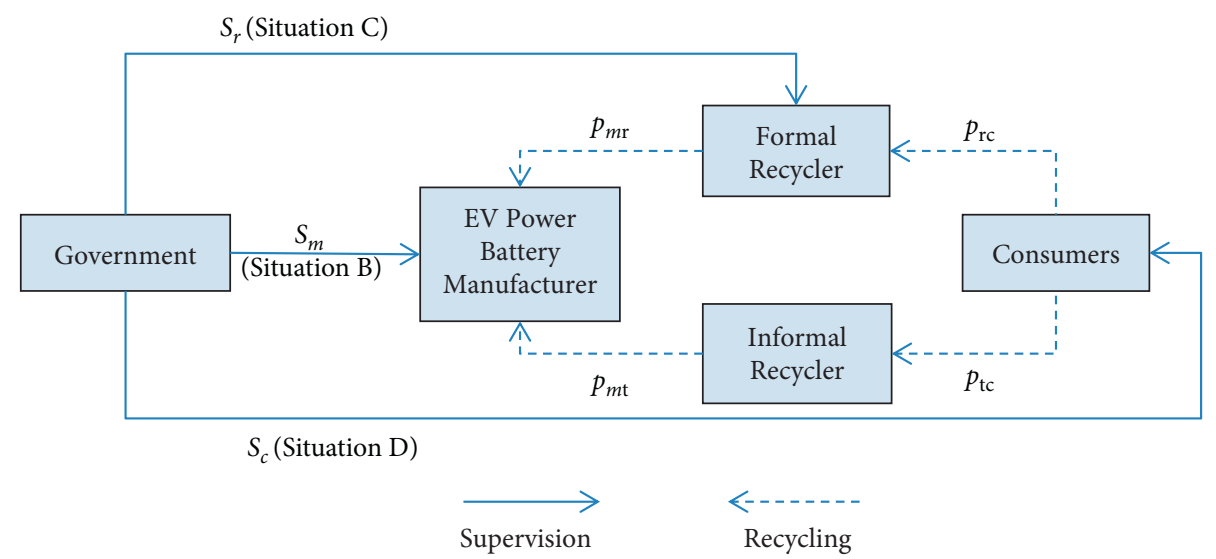

FIGURE 2: Framework of EV power battery recycling.

immutability and distributed storage among other characteristics. The data security problem of the power battery traceability system can be solved using computer technologies such as point-to-point transmission, consensus mechanism, and encryption algorithm. EV power battery manufacturer can provide technical support to the formal recycler through the traceability management platform to improve the rate of echelon use. And the government can supervise the EV power battery recycling market through the EV power battery traceability management platform and adjust the relevant mechanism, as shown in Figure 1.

In the context of $\mathrm{EV}$ power battery traceability management by blockchain technology, this study constructs a reverse supply chain comprising an EV power battery manufacturer, a power battery formal recycler, a power battery informal recycler, and consumers. Figure 2 shows the specific structure. Three government mechanisms are considered in this paper to promote the participation of each recycling subject in power battery traceability management: a reward and punishment mechanism for EV power battery manufacturers (situation $B$ ); a subsidy mechanism for formal recyclers (situation $C$ ); and a subsidy mechanism for consumers (situation $D$ ). EV power battery manufacturers have two channels to choose from: formal recycling channels and informal recycling channels. Informal recycler adopts door-to-door recycling, and formal recycler adopts the method of allowing consumers to send spent EV power batteries to stores or formal recycling stations in the community. This study assumes that $p_{r c}>p_{t c}$ because the relatively inconvenient recycling channels need to attract consumers to participate in recycling by increasing the recycling price [9]. The personnel involved in the recycling process inspect, classify, and discharge the batteries according to the information provided by the EV power battery manufacturer and the status of the actual batteries. The recycled batteries are prioritized for echelon use. Furthermore, materials are extracted from them for remanufacturing batteries that are not available for echelon use. 
TABLE 1: The description of the symbols.

\begin{tabular}{|c|c|}
\hline Parameters & Meaning \\
\hline Q & The potential market size \\
\hline$A$ & The profit of echelon use \\
\hline$B$ & The profit of extracting metal materials \\
\hline$\theta_{r}$ & The rate of echelon use of an EV power battery processed by the formal recycler \\
\hline$\theta_{t}$ & The rate of echelon use of an EV power battery processed by the informal recycler \\
\hline$k$ & Increase factor of the echelon use rate of EV power batteries participating in blockchain traceability management \\
\hline$q_{r c}$ & Number of recycled batteries from the formal recycler \\
\hline$q_{t c}$ & Number of recycled batteries from the formal recycler \\
\hline$q$ & Number of EV power batteries delivered by consumers without compensation \\
\hline$\alpha$ & Sensitivity factor of consumers to the recycling price \\
\hline$\beta$ & Recycling competition coefficient between the formal and informal recycler \\
\hline$p_{r c}$ & Recycling price per unit for the formal recycler \\
\hline$p_{t c}$ & Recycling price per unit for the informal recycler \\
\hline$p_{m r}$ & Unit transfer price paid by the power cell manufacturer to the formal recycler \\
\hline$p_{m t}$ & Unit transfer price paid by the power cell manufacturer to the informal recycler \\
\hline$c_{r}$ & Unit recycling disposal cost for the formal recycler \\
\hline$c_{t}$ & Unit recycling processing cost for the informal recycler \\
\hline$I$ & Blockchain operation cost \\
\hline$\xi_{0}$ & Minimum target recycling rate set by the government \\
\hline$S_{i}$ & $i=m, c, r$ \\
\hline$S_{m}$ & $\begin{array}{c}\text { The amount of incentives and penalties the government issues to the manufacturer participating in blockchain traceability } \\
\text { management }\end{array}$ \\
\hline$S_{c}$ & The amount of subsidy the government issues to the consumers participating in blockchain traceability management \\
\hline$S_{r}$ & The amount of subsidy the government issues to the recycler participating in blockchain traceability management \\
\hline$\Pi_{m}$ & The profit of the EV power battery manufacturer \\
\hline$\Pi_{r}$ & The profit of the formal recycler \\
\hline$\Pi_{t}$ & The profit of the informal recycler \\
\hline
\end{tabular}

3.2. Model Assumptions and Parameters' Description. We have made some assumptions about this article based on the research of related scholars:

(1) For simplification, we assume that the recycling market comprises an EV power battery manufacturer, a formal recycler, and an informal recycler [34].

(2) This study only considers the power batteries sold with EVs. Moreover, all the EV power batteries sold can be recycled. Therefore, the total supply of the EV power battery recycling market is the total supply of the EV sales market $Q$.

(3) A recycled EV power battery is a single type [22].

(4) The EV power battery traceability management platform established based on the blockchain allows only government-certified enterprises with recycling and processing qualifications to participate. This study classifies such enterprises as formal recyclers. The government cannot regulate the market transactions between the power battery manufacturer and informal recycler. Therefore, the recycling rate $\theta$ calculated from the government's perspective is the ratio of the recycling quantity $q_{r c}$ from formal recycling channels to the total supply quantity $Q$.

(5) The recycling volume under each recycling channel is affected by the recycling price. The recycling function of the informal recycler is $q_{t c}=q+\alpha p_{t c}-\beta p_{r c}$, while that of the formal recycler is $q_{r c}=\alpha p_{r c}-\beta p_{t c}$, where $\alpha>\beta>0$.

(6) The residual value of the recycled EV power battery is not 0 ; that is, $A+B>0$. To ensure the profit of each subject, let $p_{m r}>p_{r c}$ and $p_{m t}>p_{t c}$.

(7) EV power battery manufacturer as the producer bears the main responsibility of recycling, consumers are the source of recycling, and formal recyclers are the main recycling enterprises. Therefore, the initial stage can be assumed that $S_{c}>S_{r}>S_{m}$. The above rewards and penalties and subsidies can be adjusted later according to the actual situation.

(8) EV power battery manufacturer acts as the leader of Stackelberg, and the recycler modifies the strategy to be adopted according to the $\mathrm{EV}$ power battery manufacturer.

The symbols are described in the text as shown in Table 1.

\subsection{Stackelberg Game Model}

3.3.1. Mode A: Nonparticipation in Blockchain Traceability Management. In the process of EV power battery recycling, $\mathrm{EV}$ power battery manufacturer sets the transfer price of EV power batteries according to the market supply and demand situation firstly; then, the EV power battery formal recycler and informal recycler set the recycling recovery price according to the transfer price; finally, consumers pursue utility maximization and choose the recycling channel 
according to the recycling price and convenience of recycling given by each recycler. Therefore, in the case of no government mechanism intervention (situation $A$ ), the profit functions of the EV power battery manufacturer, formal recycler, and informal recycler can be expressed, respectively, as follows.

The profit function of the EV power battery manufacturer can be expressed as

$$
\begin{aligned}
\Pi_{m}^{A}= & {\left[A \theta_{r}+B\left(1-\theta_{r}\right)-p_{m r}\right]\left(\alpha p_{r c}-\beta p_{t c}\right) } \\
& +\left[A \theta_{t}+B\left(1-\theta_{t}\right)-p_{m t}\right]\left(q+\alpha p_{t c}-\beta p_{r c}\right), \\
& A \theta_{r}+B\left(1-\theta_{r}\right) .
\end{aligned}
$$

The EV power battery manufacturer needs to pay the cost of transfer $p_{m r}$ or $p_{m t}$ to the recycler and make profits from the batteries by echelon use $A$ or extracting metal materials $B$.

The profit function of the formal recycler can be expressed as

$$
\Pi_{r}^{A}=\left(p_{m r}-p_{r c}-c_{r}\right)\left(\alpha p_{r c}-\beta p_{t c}\right) .
$$

The profit function of the informal recycler can be expressed as

$$
\Pi_{t}^{A}=\left(p_{m t}-p_{t c}-c_{t}\right)\left(q+\alpha p_{t c}-\beta p_{r c}\right) .
$$

The profit of recyclers is the revenue received from the EV power battery manufacturer $p_{m r}$ or $p_{m t}$ minus the cost of recycling $p_{r c}$ or $p_{t c}$.

Proposition 1. Equation (1) is a concave function regarding $p_{m t}$ and $p_{m r}$; there exists a unique optimal solution.

Proof. The second-order partial derivatives of equation (1) for $p_{m t}$ and $p_{m r}$, respectively, give

$$
\begin{aligned}
H\left(\Pi_{m}^{A}\right) & =\left[\begin{array}{cc}
\frac{\partial^{2} \Pi_{m}^{A}}{\partial p_{m r}^{2}} & \frac{\partial^{2} \Pi_{m}^{A}}{\partial p_{m r} p_{m t}} \\
\frac{\partial^{2} \Pi_{m}^{A}}{\partial p_{m t} p_{m r}} & \frac{\partial^{2} \Pi_{m}^{A}}{\partial p_{m t}^{2}}
\end{array}\right] \\
& =\left[\begin{array}{cc}
\frac{2 \alpha\left(\beta^{2}-2 \alpha^{2}\right)}{4 \alpha^{2}-\beta^{2}} & \frac{2 \alpha^{2} \beta}{4 \alpha^{2}-\beta^{2}} \\
\frac{2 \alpha^{2} \beta}{4 \alpha^{2}-\beta^{2}} & \frac{2 \alpha\left(\beta^{2}-2 \alpha^{2}\right)}{4 \alpha^{2}-\beta^{2}}
\end{array}\right] .
\end{aligned}
$$

Because $H_{11}=\left(2 \alpha\left(\beta^{2}-2 \alpha^{2}\right) / 4 \alpha^{2}-\beta^{2}\right)<0$, det $(H)=$ $\left(4 \alpha^{2}\left(\alpha^{2}-\beta^{2}\right) / 4 \alpha^{2}-\beta^{2}\right)>0$, and then the Hessian matrix of equation (1) is negative definite; therefore, equation (1) is a concave function regarding $p_{m t}$ and $p_{m r}$; there exists a unique optimal solution.

Proposition 2. Equations (2) and (3) are concave functions regarding $p_{r c}$ and $p_{t c}$; there exists a unique optimal solution.

Proof. The first-order partial derivatives and second-order partial derivatives of equations (2) and (3) regarding $p_{r c}$ and $p_{t c}$, respectively, give

$$
\begin{aligned}
& \frac{\partial \Pi_{r}^{A}}{\partial p_{r c}}=-\alpha c_{r}+\alpha p_{m r}-2 \alpha p_{r c}+\beta p_{t c}, \\
& \frac{\partial^{2} \Pi_{r}^{A}}{\partial p_{r c}^{2}}=-2 \alpha, \\
& \frac{\partial \Pi_{t}^{A}}{\partial p_{t c}}=-q-\alpha c_{t}+\alpha p_{m t}+\beta p_{r c}-2 \alpha p_{t c}, \\
& \frac{\partial^{2} \Pi_{t}^{A}}{\partial p_{t c}^{2}}=-2 \alpha .
\end{aligned}
$$

From the partial derivatives of the above solution, $\left(\partial^{2} \Pi_{r}^{A} / \partial p_{r c}^{2}\right)=\left(\partial^{2} \Pi_{t}^{A} / \partial p_{t c}^{2}\right)=-2 \alpha<0$. Therefore, equations (2) and (3) are concave functions regarding $p_{r c}$ and $p_{t c}$, respectively: there exists a unique optimal solution.

The solution of equations (1)-(3) is solved using the inverse induction method; Appendix shows the results.

3.3.2. Mode B: Participate in Blockchain Traceability Management-Adopt a Reward and Punishment Mechanism for the Manufacturer. Spent EV power batteries need to go through steps such as dismantling and testing before being used in the echelon, and informal recyclers face many technical difficulties in processing. Through the blockchain traceability management platform, formal recycler can obtain the status information of EV power batteries and technical support provided by the battery manufacturer, which can improve the gradient utilization rate to a certain extent. The increased gradient echelon use rate is expressed in terms of $k$; the utilization rate is $\left(k+\theta_{r}\right)$. Meanwhile, when participating in EV power battery blockchain traceability management, EV power battery manufacturer and formal recycler need to pay management costs $I$, including network costs and labor costs. Therefore, in the situation of the government adopts a reward and punishment mechanism for the EV power battery manufacturer participating in blockchain traceability management (situation $B$ ), the profit functions of the EV power battery manufacturer, formal recycler, and informal recycler can be expressed, respectively, as follows.

The profit function of the EV power battery manufacturer can be expressed as 


$$
\begin{aligned}
\Pi_{m}^{B}= & {\left[A\left(k+\theta_{r}\right)+B\left(1-\theta_{r}-k\right)-p_{m r}\right]\left(\alpha p_{r c}-\beta p_{t c}\right) } \\
& +\left[A \theta_{t}+B\left(1-\theta_{t}\right)-p_{m t}\right]\left(q+\alpha p_{t c}-\beta p_{r c}\right) \\
& +S_{m}\left(\alpha p_{r c}-\beta p_{t c}-\xi_{0} Q\right)-I .
\end{aligned}
$$

Through the blockchain traceability management platform, the government can regulate the EV power battery manufacturer and formal recycler. In situation $B$, the government gives rewards to the EV power battery manufacturer when the actual recycling quantity is greater than the target recycling quantity; on the contrary, the government gives punishments to power battery manufacturers when the actual recycling quantity is less than the target recycling quantity.

The profit function of the formal recycler can be expressed as

$$
\Pi_{r}^{B}=\left(p_{m r}-p_{r c}-c_{r}\right)\left(\alpha p_{r c}-\beta p_{t c}\right)-I .
$$

The profit function of the informal recycler can be expressed as

$$
\Pi_{t}^{B}=\left(p_{m t}-p_{t c}-c\right)_{t}\left(q+\alpha p_{t c}-\beta p_{r c}\right) .
$$

As in Propositions 1 and 2, equations (4)-(6) are solved using the inverse induction method; Appendix shows the solution results.

3.3.3. Mode C: Participate in Blockchain Traceability Management-Adopt a Subsidy Mechanism for the Formal Recycler. As situation $B$, the echelon use rate can be improved when the EV power battery manufacturer and formal recycler participate in blockchain traceability management, while they need to pay a certain management cost $I$. Therefore, in the situation of the government adopts a subsidy mechanism for formal recyclers who participate in blockchain traceability management (situation $C$ ), the profit functions of the EV power battery manufacturer, formal recycler, and informal recycler can be expressed, respectively, as follows.

The profit function of the EV power battery manufacturer can be expressed as

$$
\begin{aligned}
\Pi_{m}^{C}= & {\left[A\left(k+\theta_{r}\right)+B\left(1-\theta_{r}-k\right)-p_{m r}\right]\left(\alpha p_{r c}-\beta p_{t c}\right) } \\
& +\left[A \theta_{t}+B\left(1-\theta_{t}\right)-p_{m t}\right]\left(q+\alpha p_{t c}-\beta p_{r c}\right)-I .
\end{aligned}
$$

The profit function of the formal recyclers can be expressed as

$$
\Pi_{r}^{C}=\left(p_{m r}-p_{r c}-c_{r}+S_{r}\right)\left(\alpha p_{r c}-\beta p_{t c}\right)-I .
$$

In situation $C$, the formal recycler may receive additional government subsidies $S_{r}$.
The profit function of the informal recycler can be expressed as

$$
\Pi_{t}^{C}=\left(p_{m t}-p_{t c}-c_{t}\right)\left(q+\alpha p_{t c}-\beta p_{r c}\right) .
$$

As in Propositions 1 and 2, equations (7)-(9) are solved using the inverse induction method; Appendix shows the solution results.

3.3.4. Mode D: Participate in Blockchain Traceability Management-Adopt a Subsidy Mechanism for Consumers. As situation $B$, the echelon use rate can be improved when the EV power battery manufacturer and formal recycler participate in blockchain traceability management, while they need to pay a certain management cost $I$. Therefore, in the situation of the government adopts a subsidy mechanism for consumers who hand over spent EV power batteries to the formal recycler (situation $D$ ), the profit functions of the power battery manufacturer, formal recycler, and informal recycler can be expressed as follows, respectively.

The profit function of the EV power battery manufacturer can be expressed as

$$
\begin{aligned}
\Pi_{m}^{D}= & {\left[A\left(k+\theta_{r}\right)+B\left(1-\theta_{r}-k\right)-p_{m r}\right]\left[\alpha\left(p_{r c}+S_{c}\right)-\beta p_{t c}\right] } \\
& +\left[A \theta_{t}+B\left(1-\theta_{t}\right)-p_{m t}\right]\left[q+\alpha p_{t c}-\beta\left(p_{r c}+S_{c}\right)\right]-I .
\end{aligned}
$$

The profit function of the formal recycler can be expressed as

$$
\Pi_{r}^{D}=\left(p_{m r}-p_{r c}-c_{r}+S_{r}\right)\left[\alpha\left(p_{r c}+S_{c}\right)-\beta p_{t c}\right]-I .
$$

In situation $D$, the government gives subsidies to consumers through formal recyclers.

The profit function of the informal recycler can be expressed as

$$
\Pi_{t}^{D}=\left(p_{m t}-p_{t c}-c_{t}\right)\left(q+\alpha p_{t c}-\beta p_{r c}\right) .
$$

As in Propositions 1 and 2, equations (10)-(12) are solved using the inverse induction method; Appendix shows the solution results.

\section{Model Analysis}

4.1. Analysis of the Impact of Participation in Blockchain Traceability Management on Recycling Prices

Proposition 3. $p_{m t}^{A}=p_{m t}^{B}=p_{m t}^{C}=p_{m t}^{D}$.

Proof. According to the result of the inverse induction method of solving, we obtain

$$
p_{m t}^{A}=p_{m t}^{B}=p_{m t}^{C}=p_{m t}^{D}=\frac{-q \alpha+B \alpha^{2}-B \beta^{2}+\left(\alpha^{2}-\beta^{2}\right) c_{t}+(A-B)\left(\alpha^{2}-\beta^{2}\right) \theta_{t}}{2\left(\alpha^{2}-\beta^{2}\right)} .
$$


Proposition 3 illustrates that the transfer price paid by the EV power battery manufacturer to the informal recycler is unaffected regardless of whether the EV power battery manufacturer and formal recycler participate in blockchain traceability and the mechanism adopted by the government.

$$
\begin{aligned}
& \text { Proposition 4. When } \\
& p_{m r}^{B}>p_{m r}^{C}>p_{m r}^{D}>p_{m r}^{A} .
\end{aligned}
$$

Proof. When $(A-B) k+2 c_{r}>S_{c}$,

$$
\begin{aligned}
& p_{m r}^{B}-p_{m r}^{C}=\frac{1}{2}\left(S_{m}+S_{r}\right)>0, \\
& p_{m r}^{C}-p_{m r}^{D}=\frac{1}{2}\left(S_{c}+S_{r}\right)>0, \\
& p_{m r}^{D}-p_{m r}^{A}=\frac{1}{2}\left((A-B) k+2 c_{r}-S_{c}>0\right) .
\end{aligned}
$$

When $p_{m r}^{D}-p_{m r}^{A}=(1 / 2)\left((A-B) k+2 c_{r}-S_{c}<0\right)$

$(A-B) k+2 c_{r}<S_{c}$

Proposition 4 illustrates that, after the EV power battery manufacturer and formal recycler participate in blockchain traceability, when $(A-B) k+2 c_{r}>S_{c}$, the transfer price paid by the EV power battery manufacturer to the formal recycler is greater than the transfer price when they do not participate in blockchain traceability management, and it is greatest when incentives and penalties are adopted for the EV power battery manufacturer. This is because an increase in the transfer price by the EV power battery manufacturer can help the formal recycler to recycle EV power batteries, hence recycling more EV power batteries, avoiding penalties, and obtaining more government subsidies. When $(A-B) k+2 c_{r}<S_{c}$, the government pays more subsidies to consumers through the formal recycler. The formal recycler has a stronger competitive advantage in recycling prices and can recycle more $\mathrm{EV}$ power batteries, at which time the $\mathrm{EV}$ power battery manufacturer will appropriately reduce the transfer price paid to the formal recycler.

Proposition 5. When $\quad\left((A-B) k \alpha^{2} / 3 \alpha^{2}-\beta^{2}\right)>S_{c}$, $p_{r c}^{C}>p_{r c}^{B}>p_{r c}^{D}>p_{r c}^{A}$.

Proof. When $\left((A-B) k \alpha^{2} / 3 \alpha^{2}-\beta^{2}\right)>S_{c}$,

$$
\begin{aligned}
& p_{r c}^{C}-p_{r c}^{B}=\frac{\alpha^{2}\left(S_{r}-S_{m}\right)}{4 \alpha^{2}-\beta^{2}}>0, \\
& p_{r c}^{B}-p_{r c}^{D}=\frac{\left(3 \alpha^{2}-\beta^{2}\right) S_{c}+\alpha^{2} S_{m}}{4 \alpha^{2}-\beta^{2}}>0, \\
& p_{r c}^{D}-p_{r c}^{A}=\frac{(A-B) k \alpha^{2}-\left(3 \alpha^{2}-\beta^{2}\right) S_{c}}{4 \alpha^{2}-\beta^{2}}>0 .
\end{aligned}
$$

When $\quad\left((A-B) k \alpha^{2} / 3 \alpha^{2}-\beta^{2}\right)<S_{c}$, $p_{r c}^{D}-p_{r c}^{A}=\left((A-B) k \alpha^{2}-\left(3 \alpha^{2}-\beta^{2}\right) S_{c} / 4 \alpha^{2}-\beta^{2}\right)<0$.

Proposition 5 illustrates that, after the EV power battery manufacturer and formal recycler participate in blockchain traceability, when $\left((A-B) k \alpha^{2} / 3 \alpha^{2}-\beta^{2}\right)<S_{c}$, the recycling price paid by the formal recycler to consumers is greater than that when they do not participate in blockchain traceability management. The recycling price is greatest when the subsidy mechanism is adopted for the formal recycler. This is because the increase in the recycling price by the formal recycler will increase the competitive advantage, which can help consumers to deliver spent EV power batteries to the formal recycler and consequently receive more government subsidies. When $\left((A-B) k \alpha^{2} / 3 \alpha^{2}-\beta^{2}\right)<S_{c}$, the government pays more subsidies to consumers through the formal recycler. The formal recycler has a stronger competitive advantage in recycling price in this case. Therefore, the formal recycler can also recycle EV power batteries by appropriately reducing the recycling price paid to consumers.

Proposition 6. $p_{t c}^{D}>p_{t c}^{C}>p_{t c}^{B}>p_{t c}^{A}$.

Proof.

$$
\begin{aligned}
& p_{t c}^{D}-p_{t c}^{C}=\frac{\alpha \beta\left(S_{c}-S_{r}\right)}{8 \alpha^{2}-2 \beta^{2}}>0, \\
& p_{t c}^{C}-p_{t c}^{B}=\frac{\alpha \beta\left(S_{r}-S_{m}\right)}{8 \alpha^{2}-2 \beta^{2}}>0, \\
& p_{t c}^{B}-p_{t c}^{A}=\frac{\alpha \beta\left((A-B) k+S_{m}\right)}{8 \alpha^{2}-2 \beta^{2}}>0 .
\end{aligned}
$$

Proposition 6 illustrates that, after the EV power battery manufacturer and formal recycler participate in blockchain traceability, the recycling price paid by the informal recycler to consumers is greater than the recycling price when they do not participate in blockchain traceability management. The recycling price paid to consumers is greatest when a subsidy mechanism is adopted by consumers. At this time, the formal recycler has a stronger competitive advantage. Therefore, the informal recycler must increase the recycling price to be more competitive and further obtain more EV power batteries.

4.2. Analysis of the Impact of Participation in Blockchain Traceability Management on the Number of Recycling

Proposition 7. $q_{r c}^{D}>q_{r c}^{C}>q_{r c}^{B}>q_{t c}^{A} ; q_{t c}^{A}>q_{t c}^{B}>q_{t c}^{C}>q_{t c}^{D}$.

Proof. 
TABLE 2: Summary of the values of the parameters.

\begin{tabular}{lcccccccccccccccc}
\hline Parameter & $Q$ & $A$ & $B$ & $\theta_{r}$ & $\theta_{t}$ & $k$ & $\alpha$ & $\beta$ & $q$ & $c_{r}$ & $c_{t}$ & $I$ & $\xi_{0}$ & $S_{m}$ & $S_{r}$ & $S_{c}$ \\
\hline Value & 111,509 & 11,388 & 1541 & 0.56 & 0.3 & 0.14 & 3.1 & 1.2 & 500 & 340 & 200 & 101,257 & 0.2 & 1500 & 1700 & 2000 \\
\hline
\end{tabular}

TABLE 3: The profit of each recycling subject.

\begin{tabular}{lccc}
\hline & $\Pi_{m}$ & $\Pi_{r}$ & $\Pi_{t}$ \\
\hline Situation $A$ & $1.99 \times 10^{7}$ & $6.13 \times 10^{6}$ & $1.68 \times 10^{6}$ \\
Situation $B$ & $1.99 \times 10^{6}$ & $1.35 \times 10^{7}$ & $1.08 \times 10^{6}$ \\
Situation $C$ & $3.68 \times 10^{7}$ & $2.86 \times 10^{6}$ & $1.05 \times 10^{6}$ \\
Situation $D$ & $3.88 \times 10^{7}$ & $1.51 \times 10^{7}$ & $9.95 \times 10^{5}$ \\
\hline
\end{tabular}

$$
\begin{aligned}
& q_{r c}^{D}-q_{r c}^{C}=\frac{\alpha\left(2 \alpha^{2}-\beta^{2}\right)\left(S_{c}-S_{r}\right)}{8 \alpha^{2}-2 \beta^{2}}>0, \\
& q_{r c}^{C}-q_{r c}^{B}=\frac{\alpha\left(2 \alpha^{2}-\beta^{2}\right)\left(S_{r}-S_{m}\right)}{8 \alpha^{2}-2 \beta^{2}}>0, \\
& q_{r c}^{B}-q_{r c}^{A}=\frac{\alpha\left(2 \alpha^{2}-\beta^{2}\right)\left((A-B) k+S_{m}\right)}{8 \alpha^{2}-2 \beta^{2}}>0, \\
& q_{t c}^{A}-q_{t c}^{B}=\frac{\alpha^{2} \beta\left((A-B) k+S_{m}\right)}{8 \alpha^{2}-2 \beta^{2}}>0, \\
& q_{t c}^{B}-q_{t c}^{C}=\frac{\alpha^{2} \beta\left(S_{r}-S_{m}\right)}{8 \alpha^{2}-2 \beta^{2}}>0, \\
& q_{t c}^{C}-q_{t c}^{D}=\frac{\alpha^{2} \beta\left(S_{c}-S_{r}\right)}{8 \alpha^{2}-2 \beta^{2}}>0 .
\end{aligned}
$$

Proposition 7 illustrates that, after the EV power battery manufacturer and formal recycler participate in blockchain traceability, the formal recycler obtains more EV power batteries than when they do not participate in blockchain traceability management, while the informal recycler does the opposite. Furthermore, the formal recycler obtains the most EV power batteries, and the informal recycler obtains the least EV power batteries when the subsidy mechanism is adopted for consumers. Therefore, the participation of the EV power battery manufacturer and formal recycler in blockchain traceability helps promote the flow of retired EV power batteries to the formal recycling market and reduces black market transactions. The results are optimal when the government adopts the subsidy mechanism for consumers.

Considering that the profit of each recycling subject is complex, it cannot be analyzed directly using the method of analyzing profit expressions. Therefore, it is compared after solving in the arithmetic part.

\section{Numerical Analysis}

5.1. Numerical Example. In this study, taking the Beijing New Energy Vehicle (BJEV) as an example, according to the Annual Production and Sales Snapshot of BJEV (2018-2020, three-year average sales), the potential market size $Q$ is assumed to be 111,509 . According to [16, 22, 23, 33], we obtained the relevant data of the EV power battery recycling shown in Table 2.

Table 3 shows the profits of the EV power battery manufacturer, formal recycler, and informal recycler under different scenarios.

As shown in Table 3, $\Pi_{m}^{D}>\Pi_{m}^{C}>\Pi_{m}^{A}>\Pi_{m}^{B}$, $\Pi_{r}^{D}>\Pi_{r}^{B}>\Pi_{r}^{A}>\Pi_{r}^{C}$, and $\Pi_{t}^{A}>\Pi_{t}^{B}>\Pi_{t}^{C}>\Pi_{t}^{D}$.

The profit of the EV power battery manufacturer after participating in blockchain traceability management is smaller when the government adopts the reward and punishment mechanism for the manufacturer than when they do not participate in blockchain traceability management. The profit is, however, larger when the government adopts the subsidy mechanism for consumers and the subsidy mechanism for the formal recycler than when they do not participate in blockchain traceability management. The profit is the largest when the government adopts the subsidy mechanism for consumers.

The profit of the formal recycler after participating in blockchain traceability management is smaller when the government adopts the subsidy mechanism for the formal recycler than when they do not participate in blockchain traceability management. The profit is, however, larger when the government adopts the subsidy mechanism for consumers and the reward and punishment mechanism for the EV power battery manufacturer than when they do not participate in blockchain traceability management. It is the largest when the government adopts the subsidy mechanism for consumers.

The profit of the informal recycler after each recycling subject participates in blockchain traceability management is smaller than when they do not participate in blockchain traceability management. The profit of the informal recycler is smaller than when they do not participate in blockchain traceability management; it is the smallest when the government adopts a subsidy mechanism for consumers.

In summary, it is evident that when the government adopts a subsidy mechanism for consumers, the EV power battery manufacturer and formal recycler realize the largest profits, while the informal recycler realizes the smallest profit. This promotes the participation of the EV power battery manufacturer and formal recycler in $\mathrm{EV}$ power battery blockchain traceability management.

5.2. Sensitivity Analysis. When the subsidy mechanism is adopted for the formal recycler and the subsidy mechanism is adopted for consumers, the expressions of the relationship between the profit of the EV power battery manufacturer $\Pi_{m}^{C}$ and government subsidy $S_{r}$ and those between the profit of the power battery manufacturer $\Pi_{m}^{D}$ and government subsidy $S_{c}$ are similar (see Appendix). Therefore, the images reflected in Figure 3 overlap. 


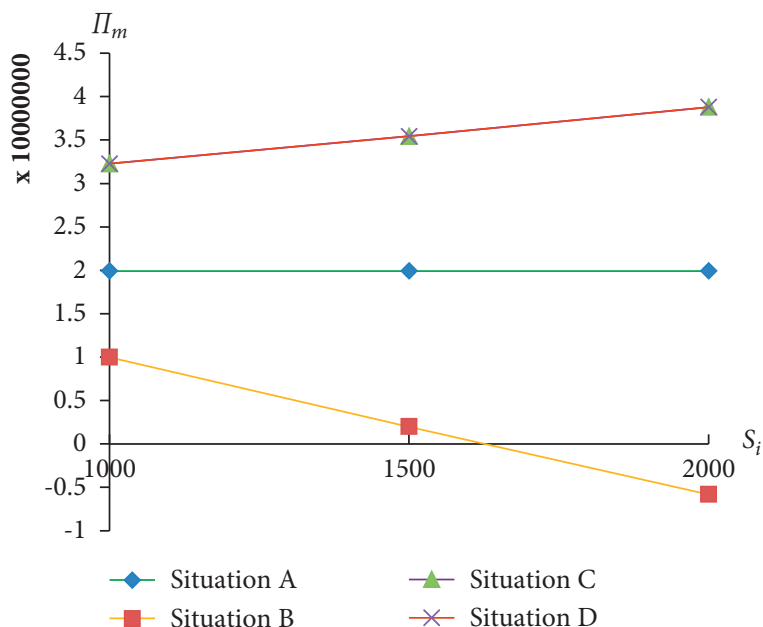

FIGURE 3: Impact of government mechanisms on the profits of the power cell manufacturer.

The expressions of the relationship between the profit $\Pi_{r}^{B}$ of the formal recycler and the government subsidy $S_{m}$ and the expressions of the relationship between the profit $\Pi_{r}^{D}$ of the formal recycler and the government subsidy $S_{c}$ are similar when the incentive and punishment mechanism is adopted for the EV power battery manufacturer and the subsidy mechanism is adopted for the consumer (see Appendix). Therefore, the images reflected in Figure 4 overlap.

The expression of the relationship between the profit of informal recyclers $\Pi_{t}^{B}$ and government subsidy $S_{m}$, the expression of the relationship between the profit of formal recyclers $\Pi_{t}^{C}$ and government subsidy $S_{r}$, and the expression of the relationship between the profit of the formal recycler $\Pi_{t}^{D}$ and government subsidy $S_{c}$ are similar when the incentive and punishment mechanism is adopted for the EV power battery manufacturer, the subsidy mechanism is adopted for formal recyclers, and the subsidy mechanism is adopted for consumers (see Appendix). Therefore, the images reflected in Figure 5 overlap.

Figures 3-5 show that, after each recycling subject joins the blockchain traceability management, when the reward and punishment mechanism is adopted for EV power battery manufacturers, the profits of the EV power battery manufacturer and formal recycler are positively proportional to the amount of reward and punishment. The profit of the informal recycler, however, is inversely proportional to the amount of reward and punishment. When the subsidy mechanism is adopted for the formal recycler, the profit of the EV power battery manufacturer is positively proportional to the amount of subsidy, and the profits of formal and informal recyclers are inversely proportional to the amount of subsidy. When the subsidy mechanism is adopted for consumers, the profits of the EV power battery manufacturer and formal recycler are proportional to the amount of rewards and penalties. Moreover, the profit of the informal recycler is inversely proportional to the amount of rewards and penalties.

In summary, when the subsidy mechanism is adopted for formal recyclers, increasing the subsidy amount reduces the

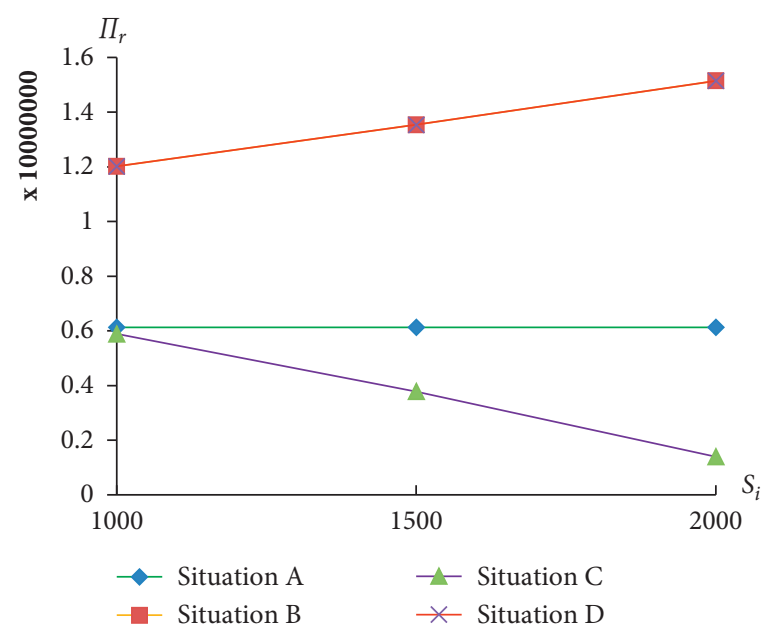

FIGURE 4: Impact of government mechanisms on the profits of the formal recycler.

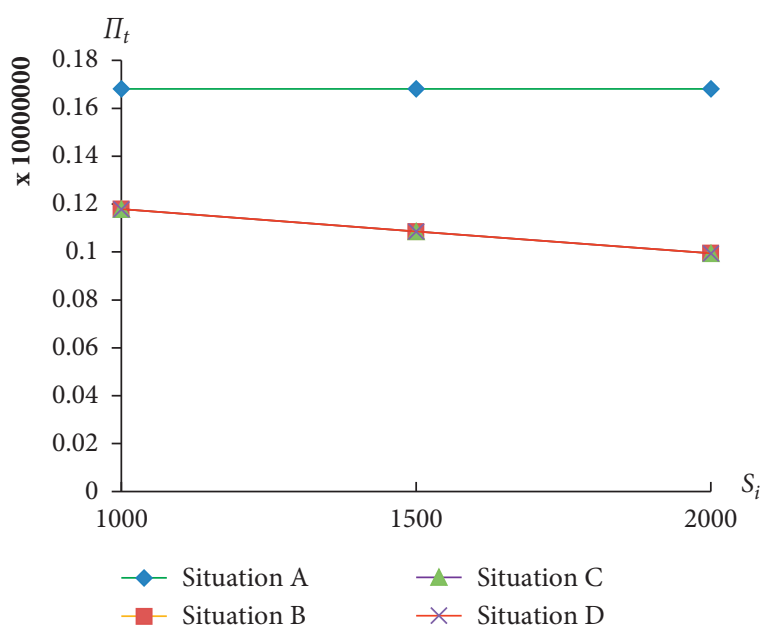

FIGURE 5: Impact of government mechanisms on the profits of the informal recycler.

profits of formal recyclers, which does not promote the participation of the formal recycler in blockchain traceability management and affects the normal implementation of EV power battery traceability management. However, increasing the amount of rewards and punishments for the EV power battery manufacturer and the amount of subsidies for consumers helps to increase the profits of the EV power battery manufacturer and formal recycler, suppressing the informal recycler and consequently reducing black market transactions and promoting the participation of each recycling subject in EV power battery blockchain traceability management; the results are optimal when consumers adopt the subsidy mechanism.

Figure 6 shows that, after each recycling subject joins blockchain traceability management, the profit of the $\mathrm{EV}$ power battery manufacturer is inversely proportional to the target recycling rate set by the government when the government adopts a reward and punishment mechanism for the EV power battery manufacturer. However, when the 


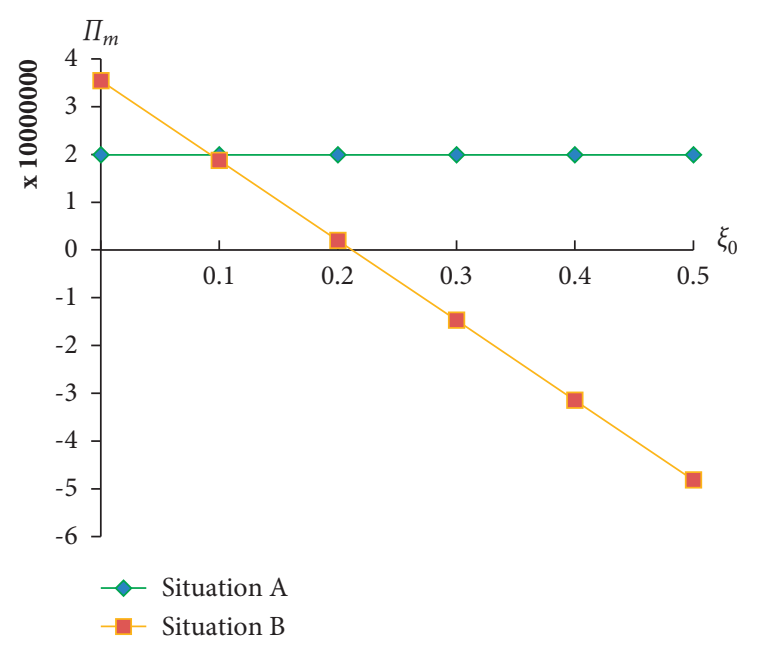

FIgURE 6: Impact of target recycling rate on the profit of the EV power battery manufacturer.

target recycling rate set by the government $\xi_{0} \geq 0.0928$, the profit of the EV power battery manufacturer after participating in blockchain traceability management is smaller than that when they do not participate in blockchain traceability management. Moreover, the profit of the EV power battery manufacturer is negative when $\xi_{0} \geq 0.212$. This is because the power battery manufacturer can only receive subsidies if their actual recycling rate is higher than the target recycling rate set by the government; otherwise, they will be penalized. Therefore, when the government adopts the reward and punishment mechanism for the EV power battery manufacturer, it should not pursue an excessively high target recycling rate at the primary stage. The government should set an appropriate target recycling rate to promote the participation of the EV power battery manufacturer in the blockchain traceability management and therefore ensure the normal implementation of the traceability management of EV power batteries. After the EV power battery manufacturer participates in blockchain traceability management, the government can adjust the target recycling rate according to the actual situation.

Figure 7 shows that the profit of EV power battery manufacturers is proportional to the increase factor of the echelon use rate. Therefore, EV power battery manufacturers can improve the echelon use rate by providing relevant technical support to EV power battery recyclers to increase the profit. When the increase factor of the echelon use rate is certain, the profit of the EV power battery manufacturer is the largest when the government adopts the subsidy mechanism for consumers, followed by the subsidy mechanism for the formal recycler. When the increase factor of the echelon use rate $k \leq 0.385$, the profit of EV power battery manufacturers when the reward and punishment mechanism is adopted is smaller than the profit when they do not participate in blockchain traceability management; when $k \leq 0.108$, the profit of EV power battery manufacturers is negative. Therefore, the enthusiasm of the manufacturer to participate in blockchain traceability management is smaller, which is not

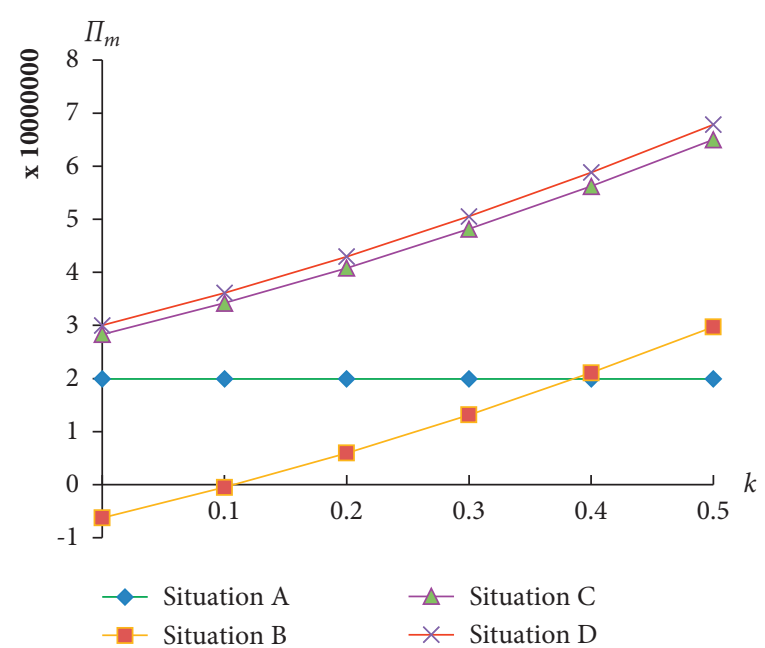

Figure 7: Effect of the increase factor of echelon use rate on the profit of the EV power battery manufacturer.

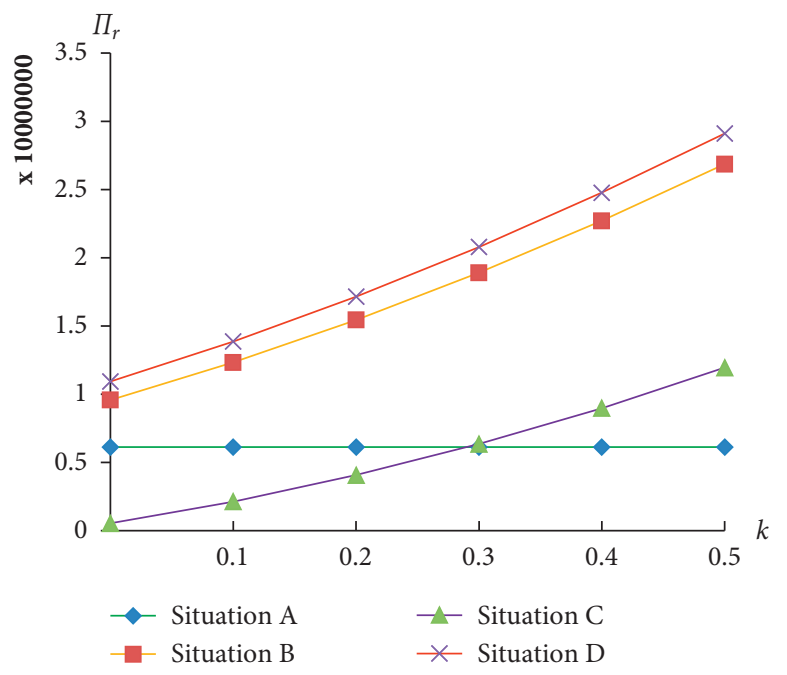

FIGURE 8: Effect of the increase factor of echelon use rate on the profit of the formal recycler.

conducive to the normal implementation of EV power battery traceability management.

Figure 8 shows that the profit of formal recyclers is proportional to the increase in the echelon use rate; therefore, the formal recycler can improve the echelon use rate by improving the recycling processing technology, hence increasing the profit. When the increase factor of the echelon use rate is certain, the profit of the formal recycler is the largest when the government adopts the subsidy mechanism for consumers, followed by the incentive and punishment mechanism for the EV power battery manufacturer. When the subsidy mechanism is adopted for the formal recycler, the profits of the formal recycler are smaller than those when they do not participate in blockchain traceability management when the echelon use rate increase factor $k<0.291$, and the enthusiasm of formal recyclers to participate in blockchain traceability 


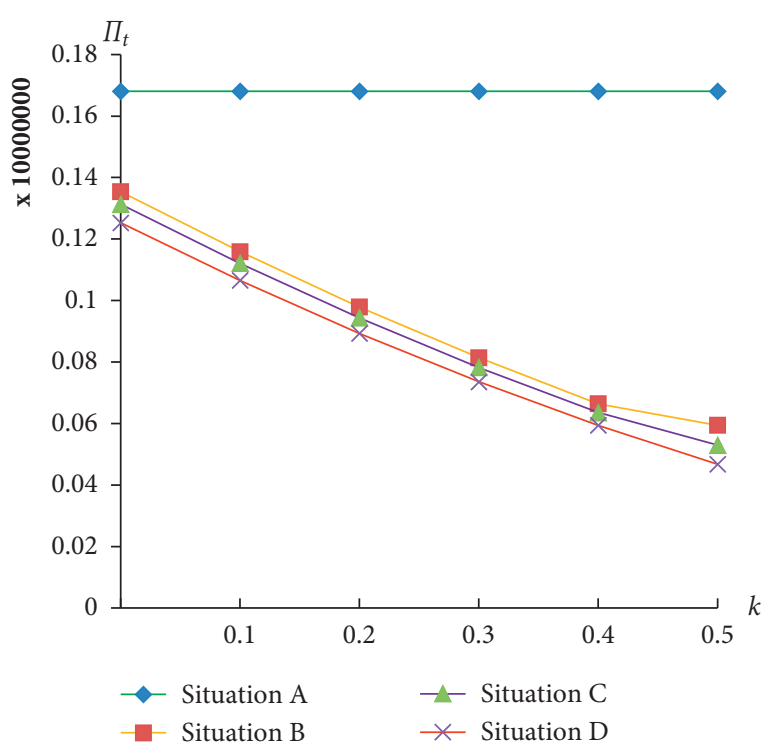

FIGURE 9: Effect of the echelon use increase factor on profits of the informal recycler.

management is smaller in this case, which is not conducive to the normal implementation of $\mathrm{EV}$ power battery traceability management.

Figure 9 shows that the profit of the informal recycler is inversely proportional to the echelon use increase factor, and the profit of the informal recycler is smaller than the profit realized when they do not participate in blockchain traceability management after each recycling subject participates in blockchain traceability management. When the echelon use rate increases by a certain factor, the profit of the informal recycler is the smallest when the government adopts a subsidy mechanism for consumers, followed by the profit when the subsidy mechanism is adopted for the formal recycler. Therefore, it can be proved that the government's adoption of mechanisms to promote the participation of each recycler in blockchain traceability management can play a role in suppressing informal recyclers, hence reducing black market transactions and promoting the transformation of the informal recycler.

\section{Conclusions}

To explore the strategies to improve the enthusiasm of EV power battery recycling subjects to participate in traceability management, this study considers factors such as the echelon use rate, government mechanism, and blockchain cost and constructs a Stackelberg game model comprising the power battery manufacturer, formal recycler, and informal recycler. Based on this model, the changes in the unit recycling price, recycling quantity, and profit of each recycling subject under different government mechanisms before and after each recycling subject participates in power battery traceability management are compared and analyzed. The main findings of this study are as follows:

(1) The participation of each recycling subject in EV power battery blockchain traceability helps improve the competitive advantage of formal recyclers, suppress informal recyclers, promote more retired power batteries into formal recycling channels and markets, and further reduce black market transactions. Additionally, the adoption of appropriate mechanisms by the government promotes the participation of each recycling subject of $\mathrm{EV}$ power batteries in blockchain traceability management, with the best result when the government adopts a subsidy mechanism for consumers.

(2) After each recycling subject participates in blockchain traceability management, the profits of the formal recycler and EV power battery manufacturer when the government adopts the reward and punishment mechanism for EV power battery manufacturers are positively proportional to the amount of government rewards and punishments, and the profits of the informal recycler are inversely proportional to the amount of government rewards and punishments. Therefore, the government increases the amount of rewards and punishments, which promotes the participation of each recycling subject in blockchain traceability management. Therefore, when the government adopts the reward and punishment mechanism for the EV power battery manufacturer, it should not pursue an excessively high target recycling rate at the primary stage. The government should set an appropriate target recycling rate to promote the participation of the EV power battery manufacturer in blockchain traceability management and consequently ensure the normal implementation of the traceability management of EV power batteries.

(3) After each recycling subject participates in blockchain traceability management, the profits of the formal recycler and EV power battery manufacturer when the government adopts the reward and punishment mechanism for formal recyclers are positively proportional to the amount of government subsidies, and the profits of the informal recycler are inversely proportional to the amount of government rewards and punishments. The profits of the formal recycler after participating in blockchain traceability management are smaller than the profits realized when they do not participate in blockchain traceability management. This does not promote the participation of formal recyclers in blockchain traceability management of EV power batteries and cannot ensure the normal implementation of traceability management of EV power batteries.

(4) The profits of power battery manufacturers and formal recyclers are positively proportional to the increase in the echelon use rate. Furthermore, the profits of informal recyclers are inversely proportional to the increase factor of the echelon use rate; therefore, the results are optimal when the subsidy mechanism is adopted for consumers. However, when the increase factor of the echelon use rate is 
small, the profit after adopting the reward and punishment mechanism for EV power battery manufacturers or adopting a subsidy mechanism for formal recyclers is smaller than the profit realized when participating in blockchain traceability management. Therefore, the government should adopt a mechanism to encourage EV power battery manufacturers to provide relevant technical support to EV power battery recyclers to improve the echelon use rate, so as to consequently increase the profit of each recycling subject and promote the participation of each recycling subject in EV power battery blockchain traceability.

In summary, in the initial stage of blockchain traceability management of EV power batteries, the government should play a leading role in establishing an institutional system that promotes the participation of various recycling subjects in the blockchain traceability management of EV power batteries. Additionally, it should set a reasonable target recycling rate and the amount of rewards and penalties, which can be adjusted according to the actual situation at a later stage. Simultaneously, formal recycling enterprises should be encouraged to adopt various ways to provide convenient recycling services for $\mathrm{EV}$ consumers, so as to prompt consumers to hand over EV power batteries to formal recycling enterprises. Under the guarantee of the government mechanism, the EV power battery recycling enterprises should actively participate in the EV power battery blockchain traceability management work to truly realize that the source of the EV power battery and the destination can be traced and consequently ensure that the spent EV power battery can be safely and environmentally friendly recycled and disposed and reused. Additionally, battery manufacturing enterprises should strengthen cooperation with formal recycling and processing enterprises, improve the echelon use rate of the spent EV power battery gradient, achieve safe recycling and environmental protection treatment, and continuously improve the EV power battery recycling industry chain.
This study has several limitations. First, for the sake of simple calculation, the EV power battery is regarded as a single type. Second, the study does not consider government and environmental benefits. Finally, the competition between multiple formal and informal recyclers is not considered. Therefore, in future research, we will consider more influencing factors and participants.

\section{Appendix}

The proofs of Propositions 1 and 2 show that there is a unique optimal solution for each profit function, so it can be solved by using the inverse induction method.

By setting $\left(\partial \Pi_{r}^{A} / \partial p_{r c}\right)=\left(p_{m r}-p_{r c}-c_{r}\right)\left(\alpha p_{r c}-\beta p_{t c}\right)=$ 0 and $\left(\partial \Pi_{t}^{A} / \partial p_{t c}\right)=\left(p_{m t}-p_{t c}-c_{t}\right)\left(q+\alpha p_{t c}-\beta p_{r c}\right)=0$ simultaneously, the following results can be obtained:

$$
\begin{aligned}
p_{r c}^{A} & =-\frac{q \beta+2 \alpha^{2} c_{r}+\alpha \beta c_{t}-2 \alpha^{2} p_{m r}-\alpha \beta p_{m t}}{4 \alpha^{2}-\beta^{2}}, \\
p_{t c}^{A} & =\frac{\alpha\left(-2 q-\beta c_{r}-2 \alpha c_{t}+\beta p_{m r}+2 \alpha p_{m t}\right)}{4 \alpha^{2}-\beta^{2}} .
\end{aligned}
$$

Finding the first-order derivatives of $\Pi_{m}^{A}$ to $p_{m r}^{A}$ and $p_{m t}^{A}$ after substituting equation (1) into equations (A.1) and (A.2) and by setting $\left(\partial \Pi_{m}^{A} / \partial p_{m r}^{A}\right)$ and $\left(\partial \Pi_{t}^{A} / \partial p_{m t}^{A}\right)$ to zero simultaneously, the following results can be obtained:

$$
p_{m r}^{A}=\frac{B \alpha^{2}-q \beta-B \beta^{2}+\left(\alpha^{2}-\beta^{2}\right) c_{r}+(A-B)\left(\alpha^{2}-\beta^{2}\right) \theta_{r}}{2\left(\alpha^{2}-\beta^{2}\right)}
$$

$$
p_{m t}^{A}=\frac{-q \alpha+B \alpha^{2}-B \beta^{2}+\left(\alpha^{2}-\beta^{2}\right) c_{t}+(A-B)\left(\alpha^{2}-\beta^{2}\right) \theta_{t}}{2\left(\alpha^{2}-\beta^{2}\right)} .
$$

Then, substituting equations (A.3) and (A.4) into equations (A.1) and (A.2), $p_{r c}^{A}$ and $p_{t c}^{A}$ can be obtained.

$$
\begin{aligned}
& p_{r c}^{A}=\frac{\alpha\left(B(\alpha-\beta)(\alpha+\beta)(2 \alpha+\beta)+3 q\left(-2 \alpha^{2}+\beta^{2}\right)-(\alpha-\beta)(\alpha+\beta)\left(\beta c_{r}+2 \alpha c_{t}-(A-B)\left(\beta \theta_{r}+2 \alpha \theta_{t}\right)\right)\right)}{2\left(4 \alpha^{4}-5 \alpha^{2} \beta^{2}+\beta^{4}\right)}, \\
& p_{t c}^{A}=\frac{\alpha\left(B(\alpha-\beta)(\alpha+\beta)(2 \alpha+\beta)+3 q\left(-2 \alpha^{2}+\beta^{2}\right)-(\alpha-\beta)(\alpha+\beta)\left(\beta c_{r}+2 \alpha c_{t}-(A-B)\left(\beta \theta_{r}+2 \alpha \theta_{t}\right)\right)\right)}{2\left(4 \alpha^{4}-5 \alpha^{2} \beta^{2}+\beta^{4}\right)} .
\end{aligned}
$$


By substituting equations (A.5) and (A.6) into $q_{t c}=q+$ $\alpha p_{t c}-\beta p_{r c}$ and $q_{r c}=\alpha p_{r c}-\beta p_{t c}, \quad q_{r c}^{A}$ and $q_{t c}^{A}$ can be obtained.

$$
\begin{aligned}
& q_{r c}^{A}=\frac{\alpha\left(2 B \alpha^{2}+q \beta-B \alpha \beta-B \beta^{2}+\left(-2 \alpha^{2}+\beta^{2}\right) c_{r}+\alpha \beta c_{t}+2 A \alpha^{2} \theta_{r}-2 B \alpha^{2} \theta_{r}-A \beta^{2} \theta_{r}+B \beta^{2} \theta_{r}-A \alpha \beta \theta_{t}+B \alpha \beta \theta_{t}\right)}{8 \alpha^{2}-2 \beta^{2}} \\
& q_{t c}^{A}=\frac{\alpha\left(2 q \alpha+2 B \alpha^{2}-B \alpha \beta-B \beta^{2}+\alpha \beta c_{r}+\left(-2 \alpha^{2}+\beta^{2}\right) c_{t}-A \alpha \beta \theta_{r}+B \alpha \beta \theta_{r}+2 A \alpha^{2} \theta_{t}-2 B \alpha^{2} \theta_{t}-A \beta^{2} \theta_{t}+B \beta^{2} \theta_{t}\right)}{8 \alpha^{2}-2 \beta^{2}}
\end{aligned}
$$

Substituting equations (A.3)-(A.8) into equations (1)-(3), respectively, we can obtain the optimal solution for each subject's profit.

$$
\begin{aligned}
\Pi_{m}^{A}= & \frac{1}{4\left(4 \alpha^{4}-5 \alpha^{2} \beta^{2}+\beta^{4}\right)} \alpha\left(\left(-q \beta+B\left(-\alpha^{2}+\beta^{2}\right)\right)+(\alpha-\beta)(\alpha+\beta)\left(c_{r}+(-A+B) \theta_{r}\right)\right) \\
& \cdot\left(-q \beta+B\left(-2 \alpha^{2}+\alpha \beta+\beta^{2}\right)+\left(2 \alpha^{2}-\beta^{2}\right) c_{r}-\alpha \beta c_{t}-(A+B)\left(\left(2 \alpha^{2}-\beta^{2}\right) \theta_{r}-\alpha \beta \theta_{t}\right)\right) \\
& +\left(q \alpha+B(\alpha-\beta)(\alpha+\beta)-(\alpha-\beta)(\alpha+\beta)\left(c_{t}+(-A+B) \theta_{t}\right)\right) \\
& \cdot\left(2 q \alpha+B(\alpha-\beta)(2 \alpha+\beta)+\alpha \beta c_{r}+\left(2 \alpha^{2}-\beta^{2}\right) c_{t}+(A-B)\left(-\alpha \beta \theta_{r}+\left(2 \alpha^{2}-\beta^{2}\right) \theta_{t}\right)\right) .
\end{aligned}
$$

Similarly, the optimal solutions in other situations can be obtained.

$$
\begin{aligned}
\Pi_{r}^{A}= & \frac{\alpha\left(q \beta+B(\alpha-\beta)(2 \alpha+\beta)+\left(-2 \alpha^{2}+\beta^{2}\right) c_{r}+\alpha \beta c_{t}+(A-B)\left(\left(2 \alpha^{2}-\beta^{2}\right) \theta_{r}-\alpha \beta \theta_{t}\right)\right)^{2}}{4\left(-4 \alpha^{2}+\beta^{2}\right)^{2}}, \\
\Pi_{t}^{A}= & \frac{\alpha\left(-2 \alpha(q+B \alpha)+B \alpha \beta+B \beta^{2}-\alpha \beta c_{r}+\left(2 \alpha^{2}-\beta^{2}\right) c_{t}-(A-B)\left(-\alpha \beta \theta_{r}+\left(2 \alpha^{2}-\beta^{2}\right) \theta_{t}\right)\right)^{2}}{4\left(-4 \alpha^{2}+\beta^{2}\right)^{2}}, \\
\Pi_{m}^{B}= & \frac{1}{4}\left(\frac{1}{4 \alpha^{4}-5 \alpha^{2} \beta^{2}+\beta^{4}} \alpha\left(-q \beta+(B(-1+k)-A k)(\alpha-\beta)(\alpha+\beta)+(\alpha-\beta)(\alpha+\beta)\left(c_{r}+S_{m}+(-A+B) \theta_{r}\right)\right)\right. \\
& \left.(2(B(-1+k)-A k)) \alpha^{2}-q B+B \alpha \beta+(B+A k-B k) \beta^{2}+\left(2 \alpha^{2}-\beta^{2}\right) c_{r}-\alpha \beta c_{t}-\left(2 \alpha^{2}-\beta^{2}\right)\left(S_{m}+(A-B) \theta_{r}\right)+(A-B) \alpha \beta \theta_{t}\right) \\
& +\frac{1}{4 \alpha^{4}-5 \alpha^{2} \beta^{2}+\beta^{4}} \alpha\left(-2 \alpha(q+B \alpha)+(B+A k-B k) \alpha \beta+B \beta^{2}-\alpha \beta c_{r}+\left(2 \alpha^{2}-\beta^{2}\right) c_{t}\right. \\
& \left.+\alpha \beta\left(S_{m}+(A-B) \theta_{r}\right)-(A-B)\left(2 \alpha^{2}-\beta^{2}\right) \theta_{t}\right)\left(-\alpha(q+B \alpha)+B \beta^{2}+(\alpha-\beta)(\alpha+\beta)\left(c_{t}+(-A-B) \theta_{t}\right)\right) \\
& -4 I_{m}+\frac{1}{4 \alpha^{2}-\beta^{2}} 2 S_{m}\left(\alpha \left(2(B+A k-B k) \alpha^{2}+(q-B \alpha) \beta+(B(-1+k)-A k) \beta^{2}+\left(-2 \alpha^{2}+\beta^{2}\right) c_{r}+\alpha \beta c_{t}\right.\right. \\
& \left.\left.\left.+\left(2 \alpha^{2}-\beta^{2}\right)\left(S_{m}+(A-B) \theta_{r}\right)+(-A+B) \alpha \beta \theta_{t}\right)+2 Q\left(-4 \alpha^{2}-\beta^{2}\right) \xi_{0}\right)\right)
\end{aligned}
$$




$$
\begin{aligned}
& \Pi_{r}^{B}=\frac{1}{4\left(-4 \alpha^{2}+\beta^{2}\right)^{2}} \alpha\left(2(B(-1+k)-A k) \alpha^{2}-q \beta+B \alpha \beta+(B+A k-B k) \beta^{2}+\left(-2 \alpha^{2}+\beta^{2}\right) c_{r}-\alpha \beta c_{t}-2 \alpha^{2} S_{m}+\beta^{2} S_{m}\right. \\
& \left.-2 A \alpha^{2} \theta_{r}+2 B \alpha^{2} \theta_{r}+A \beta^{2} \theta_{r}-B \beta^{2} \theta_{r}+(A-B) \alpha \beta \theta_{t}\right) \\
& \cdot\left(2(B(-1+k)-A k) \alpha^{2}-q \beta+B \alpha \beta+(B+A k-B k) \beta^{2}+\left(2 \alpha^{2}-\beta^{2}\right) c_{r}-\alpha \beta c_{t}-\left(2 \alpha^{2}-\beta^{2}\right)\right) \\
& \cdot\left(S_{m}+(A-B) \theta_{r}+(A-B) \alpha \beta \theta_{t}\right)-I_{r} \text {, } \\
& \Pi_{t}^{B}=\frac{\alpha\left(-2 \alpha(q+B \alpha)+(B+A k-B k) \alpha \beta+B \beta^{2}-\alpha \beta c_{r}+\left(-2 \alpha^{2}-\beta^{2}\right) c_{t}+\alpha \beta\left(S_{m}+(A-B) \theta_{r}\right)-(A-B)\left(2 \alpha^{2}-\beta^{2}\right) \theta_{t}\right)^{2}}{4\left(-4 \alpha^{2}+\beta^{2}\right)^{2}}, \\
& \Pi_{m}^{C}=\frac{1}{4}\left(\frac{1}{4 \alpha^{4}-5 \alpha^{2} \beta^{2}+\beta^{4}} \alpha\left(-q \beta+(B(-1+k)-A k)(\alpha-\beta)(\alpha+\beta)+(\alpha-\beta)(\alpha+\beta)\left(c_{r}-S_{r}+(-A+B) \theta_{r}\right)\right)\right. \\
& \cdot\left(2(B(-1+k)-A k) \alpha^{2}-q \beta+B \alpha \beta+(B+A k-B k) \beta^{2}+\left(2 \alpha^{2}-\beta^{2}\right) c_{r}-\alpha \beta c_{t}-\left(2 \alpha^{2}-\beta^{2}\right)\right. \\
& \left.\cdot\left(S_{r}+(A-B) \theta_{r}\right)+(A-B) \alpha \beta \theta_{t}\right) \\
& +\frac{1}{4 \alpha^{4}-5 \alpha^{2} \beta^{2}+\beta^{4}} \alpha\left(\left(-2 \alpha(q+B \alpha)+(B+A k-B k) \alpha \beta+B \beta^{2}-\alpha \beta c_{r}+\left(2 \alpha^{2}-\beta^{2}\right) c_{t}\right.\right. \\
& \left.\left.\left.+\alpha \beta\left(S_{r}+(A-B) \theta_{r}\right)-(A-B)\left(2 \alpha^{2}-\beta^{2}\right) \theta_{t}\right)\left(-\alpha(q+B \alpha)+B \beta^{2}+(\alpha-\beta)(\alpha+\beta)\left(c_{t}+(-A+B) \theta_{t}\right)\right)\right)-4 I_{m}\right), \\
& \Pi_{r}^{C}=\frac{1}{4\left(-4 \alpha^{2}+\beta^{2}\right)^{2}} \alpha\left(2(B(-1+k)-A k) \alpha^{2}-q \beta+B \alpha \beta+(B+A k-B k) \beta^{2}+\left(2 \alpha^{2}-\beta^{2}\right) c_{r}-\alpha \beta c_{t}\right. \\
& \left.+6 \alpha^{2} S_{r}-\beta^{2} S_{r}-2 A \alpha^{2} \theta_{r}+2 B \alpha^{2} \theta_{r}+A \beta^{2} \theta_{r}-B \beta^{2} \theta_{r}+(A-B) \alpha \beta \theta_{t}\right) \\
& \cdot\left(2(B(-1+k)-A k) \alpha^{2}-q \beta+B \alpha \beta+(B+A k-B k) \beta^{2}+\left(2 \alpha^{2}-\beta^{2}\right) c_{r}-\alpha \beta c_{t}\right. \\
& \left.-\left(2 \alpha^{2}-\beta^{2}\right)\left(S_{r}+(A-B) \theta_{r}\right)+(A-B) \alpha \beta \theta_{t}\right)-I_{r} \text {, } \\
& \Pi_{t}^{C}=\frac{\alpha\left(-2 \alpha(q+B \alpha)+(B+A k-B k) \alpha \beta+B \beta^{2}-\alpha \beta c_{r}+\left(2 \alpha^{2}-\beta^{2}\right) c_{t}+\alpha \beta\left(S_{r}+(A-B) \theta_{r}\right)-(A-B)\left(2 \alpha^{2}-\beta^{2}\right) \theta_{t}\right)^{2}}{4\left(-4 \alpha^{2}+\beta^{2}\right)^{2}}, \\
& \Pi_{m}^{D}=\frac{1}{4}\left(\frac{1}{4 \alpha^{4}-5 \alpha^{2} \beta^{2}+\beta^{4}} \alpha\left(-q \beta+(B(-1+k)-A k)(\alpha-\beta)(\alpha+\beta)+(\alpha-\beta)(\alpha+\beta)\left(c_{r}-S_{c}+(-A+B) \theta_{r}\right)\right)\right. \\
& \cdot\left(2(B(-1+k)-A k) \alpha^{2}-q \beta+B \alpha \beta+(B+A k-B k) \beta^{2}+\left(2 \alpha^{2}-\beta^{2}\right) c_{r}\right. \\
& \left.-\alpha \beta c_{t}-\left(2 \alpha^{2}-\beta^{2}\right)\left(S_{c}+(A-B) \theta_{r}\right)+(A-B) \alpha \beta \theta_{t}\right) \\
& +\frac{1}{4 \alpha^{4}-5 \alpha^{2} \beta^{2}+\beta^{4}} \alpha\left(\left(-2 \alpha(q+B \alpha)+(B+A k-B k) \alpha \beta+B \beta^{2}-\alpha \beta c_{r}+\left(2 \alpha^{2}-\beta^{2}\right) c_{t}+\alpha \beta\left(S_{c}+(A-B) \theta_{r}\right)\right.\right. \\
& \left.\left.\left.-(A-B)\left(2 \alpha^{2}-\beta^{2}\right) \theta_{t}\right)\left(-\alpha(q+B \alpha)+B \beta^{2}+(\alpha-\beta)(\alpha+\beta)\left(c_{t}+(-A+B) \theta_{t}\right)\right)\right)-4 I_{m}\right), \\
& \Pi_{r}^{D}=\frac{1}{4\left(-4 \alpha^{2}+\beta^{2}\right)^{2}} \alpha\left(2(B(-1+k)-A k) \alpha^{2}-q \beta+B \alpha \beta+(B+A k-B k) \beta^{2}+\left(2 \alpha^{2}-\beta^{2}\right) c_{r}\right. \\
& \left.-\alpha \beta c_{t}-2 \alpha^{2} S_{c}+\beta^{2} S_{c}-2 A \alpha^{2} \theta_{r}+2 B \alpha^{2} \theta_{r}+A \beta^{2} \theta_{r}-B \beta^{2} \theta_{r}+(A-B) \alpha \beta \theta_{t}\right) \\
& \cdot\left(2(B(-1+k)-A k) \alpha^{2}-q \beta+B \alpha \beta+(B+A k-B k) \beta^{2}+\left(2 \alpha^{2}-\beta^{2}\right) c_{r}-\alpha \beta c_{t}-\left(2 \alpha^{2}-\beta^{2}\right)\right. \\
& \left.\cdot\left(S_{c}+(A-B) \theta_{r}\right)+(A-B) \alpha \beta \theta_{t}\right)-I_{r} \text {, } \\
& \Pi_{t}^{D}=\frac{\alpha\left(-2 \alpha(q+B \alpha)+(B+A k-B k) \alpha \beta+B \beta^{2}-\alpha \beta c_{r}+\left(2 \alpha^{2}-\beta^{2}\right) c_{t}+\alpha \beta\left(S_{c}+(A-B) \theta_{r}\right)-(A-B)\left(2 \alpha^{2}-\beta^{2}\right) \theta_{t}\right)^{2}}{4\left(-4 \alpha^{2}+\beta^{2}\right)^{2}} \text {. }
\end{aligned}
$$




\section{Data Availability}

The simulation data used to support the findings of this study are included within this article.

\section{Conflicts of Interest}

The authors declare that there are no conflicts of interest regarding the publication of this paper.

\section{Acknowledgments}

This research was supported by the National Social Science Foundation of China (Grant no. 20BGL200).

\section{References}

[1] L. Zhen, L. Huang, and W. Wang, "Green and sustainable closed-loop supply chain network design under uncertainty," Journal of Cleaner Production, vol. 227, pp. 1195-1209, 2019.

[2] S. Council, "State council's decision on accelerating the cultivation and development of strategic emerging industry," 2010, https://www.gov.cn/zwgk/2010-10/18/content_ 1724848.htm.

[3] CAAM, "Overview of the production and sales of the automotive industry in December 2020," 2021, https://www.autostats.org.cn/ReadArticle.asp?NewsID=10926.

[4] L. Zhang, Y. Liu, B. Pang, B. Sun, and A. Kokko, "Second use value of China's new energy vehicle battery: a view based on multi-scenario simulation," Sustainability, vol. 12, no. 1, 2020.

[5] X. Zeng, J. Li, and L. Liu, "Solving spent lithium-ion battery problems in China: opportunities and challenges," Renewable and Sustainable Energy Reviews, vol. 52, pp. 1759-1767, 2015.

[6] H. Hao, "Fifth profit source: commercial value and mode of reverse logistics in China," Logistics Technology, vol. 36, no. 8, pp. 47-50, 2017.

[7] L. Zhen, "Modeling of yard congestion and optimization of yard template in container ports," Transportation Research Part B: Methodological, vol. 90, pp. 83-104, 2016.

[8] L. Zhen, Z. Liang, D. Zhuge, L. H. Lee, and E. P. Chew, "Daily berth planning in a tidal port with channel flow control," Transportation Research Part B: Methodological, vol. 106, pp. 193-217, 2017.

[9] X. Xia, Q. Zhu, and H. Wang, "Game model for end-of-life vehicles between formal recycling channels and informal recycling channel based on governmental different policies," Journal of Systems Management, vol. 24, no. 1, pp. 82-90, 2016.

[10] MIIT, "Interim regulations on the traceability management of the recycling and utilization of new energy vehicle power batteries," 2018, https://www.miit.gov.cn/jgsj/jns/gzdt/art/ 2020/art_cla708247cc54b068ea60ceaff0b044d.html.

[11] M. Kouhizadeh and J. Sarkis, "Blockchain practices, potentials, and perspectives in greening supply chains," Sustainability, vol. 10, no. 10, 2018.

[12] J. Leng, R. Guolei, P. Jiang et al., "Blockchain-empowered sustainable manufacturing and product lifecycle management in industry 4.0: a survey," Renewable and Sustainable Energy Reviews, vol. 132, 2020.

[13] R. C. Savaskan, S. Bhattacharya, and L. N. Van Wassenhove, "Closed-loop supply chain models with product remanufacturing," Management Science, vol. 50, no. 2, pp. 239-252, 2004.
[14] H. Liu, M. Lei, H. Deng, G. Keong Leong, and T. Huang, “A dual channel, quality-based price competition model for the WEEE recycling market with government subsidy," Omega, vol. 59, pp. 290-302, 2016.

[15] Y. Li, F. Xu, and X. Zhao, "Governance mechanisms of dualchannel reverse supply chains with informal collection channel," Journal of Cleaner Production, vol. 155, pp. 125-140, 2017.

[16] Y. Tang, Q. Zhang, Y. Li, G. Wang, and Y. Li, "Recycling mechanisms and policy suggestions for spent electric vehicles' power battery-a case of Beijing," Journal of Cleaner Production, vol. 186, pp. 388-406, 2018.

[17] H. Hao, Q. Zhang, and Z. Wang, "Feasibility evaluation for reverse logistics of end-of-life vehicles battery-based on improved fuzzy neural network," China Business and Market, vol. 32, no. 6, pp. 15-24, 2018.

[18] W. Wang and Y. Wu, "An overview of recycling and treatment of spent LiFePO 4 batteries in China," Resources, Conservation and Recycling, vol. 127, pp. 233-243, 2017.

[19] A. Beaudet, F. Larouche, K. Amouzegar, P. Bouchard, and K. Zaghib, "Key challenges and opportunities for recycling electric vehicle battery materials," Sustainability, vol. 12, no. $14,2020$.

[20] H. Hao, J. Zhang, and Q. Zhang, "Development strategy of power battery recycling reverse logistics under circular economy in China," Ecological Economy, vol. 36, no. 1, pp. 86-91, 2020.

[21] J. Li, Z. Wang, B. Jiang, and T. Kim, "Coordination strategies in a three-echelon reverse supply chain for economic and social benefit," Applied Mathematical Modelling, vol. 49, pp. 599-611, 2017.

[22] X. Gu, P. Ieromonachou, L. Zhou, and M.-L. Tseng, "Developing pricing strategy to optimise total profits in an electric vehicle battery closed loop supply chain," Journal of Cleaner Production, vol. 203, pp. 376-385, 2018.

[23] Y. Tang, Q. Zhang, Y. Li, H. Li, X. Pan, and B. McLellan, "The social-economic-environmental impacts of recycling retired EV batteries under reward-penalty mechanism," Applied Energy, vol. 251, 2019.

[24] L. Wang, X. Wang, and W. Yang, "Optimal design of electric vehicle battery recycling network-from the perspective of electric vehicle manufacturers," Applied Energy, vol. 275, 2020.

[25] Z. Wang, H. Hao, F. Gao, Q. Zhang, J. Zhang, and Y. Zhou, "Multi-attribute decision making on reverse logistics based on DEA-TOPSIS: a study of the Shanghai end-of-life vehicles industry," Journal of Cleaner Production, vol. 214, pp. 730737, 2019.

[26] H. Hao, J. Zhang, Q. Zhang, L. Yao, and Y. Sun, "Improved gray neural network model for healthcare waste recycling forecasting," Journal of Combinatorial Optimization, vol. 39, pp. 1-18, 2019.

[27] K. Dandage, R. Badia-Melis, and L. Ruiz-García, "Indian perspective in food traceability: a review," Food Control, vol. 71, pp. 217-227, 2017.

[28] Y. P. Tsang, K. L. Choy, C. H. Wu, G. T. S. Ho, and H. Y. Lam, "Blockchain-driven IoT for food traceability with an integrated consensus mechanism," IEEE Access, vol. 7, pp. 129000-129017, 2019.

[29] Q. Lin, H. Wang, X. Pei, and J. Wang, "Food safety traceability system based on blockchain and EPCIS," IEEE Access, vol. 7, pp. 20698-20707, 2019.

[30] M. P. Caro, M. S. Ali, M. Vecchio, and R. Giaffreda, "Blockchain-based traceability in Agri-Food supply chain 
management: a practical implementation," in Proceedings of the 2018 IoT Vertical and Topical Summit on Agriculture-Tuscany (IOT Tuscany), Tuscany, Italy, May 2018.

[31] H. Feng, X. Wang, Y. Duan, J. Zhang, and X. Zhang, “Applying blockchain technology to improve agri-food traceability: a review of development methods, benefits and challenges," Journal of Cleaner Production, vol. 260, 2020.

[32] S. Gong, P. Ding, X. Yan, Y. Wang, and B. Huang, "Design of power battery data monitoring and sharing system based on blockchain," in Proceedings of the 2019 3rd International Conference on Electronic Information Technology and Computer Engineering (EITCE), pp. 830-833, IEEE, Xiamen, China, October 2019.

[33] P. K. Gopalakrishnan, J. Hall, and S. Behdad, "Cost analysis and optimization of blockchain-based solid waste management traceability system," Waste Management, vol. 120, pp. 594-607, 2021.

[34] L. Shao, J. Yang, and M. Zhang, "Subsidy scheme or price discount scheme? mass adoption of electric vehicles under different market structures," European Journal of Operational Research, vol. 262, no. 3, pp. 1181-1195, 2017. 